\title{
A Link-state QoS Routing Protocol for Ad Hoc Networks
}

\author{
Anelise Munaretto $^{1} \quad$ Hakim Badis $^{2} \quad$ Khaldoun Al Agha $^{2} \quad$ Guy Pujolle $^{1}$ \\ ${ }^{1}$ LIP6 Laboratory, University of Paris VI, 8, rue du Capitaine Scott, 75015, Paris, France \\ \{Anelise.Munaretto, Guy.Pujolle\} @lip6.fr \\ ${ }^{2}$ LRI Laboratory, University of Paris XI - INRIA, Bât 490, 91405, Orsay, France \\ \{Hakim.Badis, Khaldoun.Alagha\}@1ri.fr
}

\begin{abstract}
A Quality-of-Service (QoS) routing protocol is developed for mobile Ad Hoc Networks. We perform the proposed QoS-based routing in the Optimized Link State Routing (OLSR) protocol, introducing more appropriate metric than the hop distance. In our simulations the QoS routing protocol produces better performance comparing with the besteffort OLSR protocol.
\end{abstract}

Keywords: MANET; QoS routing; OLSR; delay and bandwidth metrics; admission control.

\section{INTRODUCTION}

Addressing QoS support in the Internet has been widely investigated [1]. Nevertheless, such previous efforts find limited application to Mobile Ad Hoc Networks (MANETs) because of bandwidth constraints and dynamic network topology of MANETs. To support QoS, the link characteristics such as delay, bandwidth, cost, loss rate, and error rate in the network should be available and manageable. However, obtaining and managing the link characteristics in MANETs is a challenging task because the characteristic of a wireless link change due to resource limitations and mobility of hosts [2].

Therefore, routing protocols in ad hoc networks must be adaptive to face frequent topology changes because of node mobility. Such frequent changes render the available state information outdated. Many protocols have been proposed to address such issue [3]. The number of hops is the most common criteria adopted by the routing protocols proposed in the MANET Working Group in IETF. In an ad hoc network, the communication is done over wireless media. In that case, the number of hops criteria is not necessarily the most suitable metric to be adopted by a routing decision. In such a media, a multi-criteria proactive routing protocol allowing for the network characteristics becomes more appropriated to best select one route among multiple available paths. Route selection must take into account the current conditions of the links. The basic function of QoS routing is to find a network path which satisfies the given constraints. In addition, most QoS routing algorithms consider the optimization of resource utilization.

The link state routing approach makes available detailed information about the connectivity and conditions found in the network; and hence increases the chances that a node will be able to generate a route that meets a specified set of requirement constraints.

This paper aims at specifying a Link-state QoS Routing Protocol for Ad Hoc Networks. We propose to implement QoS functionality to deal with limited available resources in a dynamic environment.

For instance, to analyze the performance of the proposed QoS-based routing, we add the consideration of the QoS requirements of flows to the Optimized Link State Routing (OLSR) protocol [4], which already considers the hop distance.

\section{OPtimized LinK State Routing (OLSR) PROTOCOL}

OLSR is a proactive routing protocol for mobile ad hoc networks. Such protocol is adopted for the reason that it reduces the size of the control messages and minimizes the overhead from the flooding of control traffic. The protocol inherits the stability of a link state algorithm and has the advantage of having routes immediately available when needed due to its proactive nature. OLSR is an optimization over the classical link state protocol, tailored for mobile ad hoc networks.

The OLSR operates as a table driven and proactive protocol regularly exchanging topology information with other nodes of the network. The key concept used in the protocol is that of multipoint relays (MPRs). MPRs are selected nodes which forward broadcast messages during the flooding process. The idea of MPR is to minimize the overhead of flooding messages in the network by reducing duplicate retransmissions in the same region. Each node in the network selects a set of nodes in its symmetric neighborhood which 
may retransmit its messages. Each node selects its MPR set among its one hop symmetric neighbors. This set is selected such that it covers (in terms of radio range) all nodes that are two hops away.

The nodes selected as a MPR by some of the neighbor nodes, announce periodically in their control messages their condition of MPR to their neighborhood. Thereby, a node announces to the network, that it has reachability to the nodes, which have selected it as MPR. In route calculation, the MPRs are used to form the route from a given node to any destination in the network. The protocol uses the MPRs to facilitate the efficient flooding of control messages in the network. A node selects its MPR among its one-hop neighbors with symmetric link. Therefore, selecting the route through MPRs automatically avoids the problems associated with data packet transfer over unidirectional links. Each node maintains information about the neighbors that have selected it as MPR. A node obtains such information from periodic control messages received from the neighbors.

\section{USING THE DISCOVERY MECHANISM OF OLSR TO SETUP QOS ROUTES}

Given the requirements to establish a session, a QoS routing protocol needs to find a route with sufficient bandwidth. This is not easy, because even to find out the maximum available bandwidth along a given route is NPcomplete. A recently work [5] proposes an efficient algorithm for calculating the end-to-end bandwidth on a path. This paper uses such algorithm together with the route discovery mechanism of AODV [6] to setup QoS routes. The QoS routing protocol is compared with the original, besteffort (BE) AODV protocol. The advantage of QoS routing protocol becomes apparent when traffic gets heavy. A major criticism of such QoS routing protocol is that it is designed without considering the situation when multiple QoS routes are being setup simultaneously. Therefore, a protocol which produces multiples routes will be more appropriate than AODV.

To offer QoS, we add extensions to OLSR messages used during a neighbor discovery. We take advantage of such periodically control messages to exchange the essential information to achieve the QoS requirements. Each node should detect the interfaces from neighbors with which it has a direct and symmetric link. Uncertainties over radio propagation may make some links unidirectional. As a consequence, all links must be checked in both directions to be considered valid.

To perform neighbor detection, each node broadcasts control messages containing information about heard neighbor interfaces and their link status. Control messages are broadcasted to all one-hop neighbors and are emitted on each MANET interface of the node.

OLSR supports protocol extensions that may enhance the functionality of the original protocol. Such extensions are introduced as additions to the protocol without breaking backwards compatibility with earlier versions. Possible extensions may be sleep mode operation, multicast routing, and quality of service. Such extensions specify the QoS requirements that must be met by nodes [7].

The QoS support to the protocol is implemented by extending the routing table. To offer QoS routing, at least two parameters must be considered: minimum available bandwidth and maximum delay. Each node maintains a routing table to route data destined to the other nodes in the network. The routing table is based on the information contained in the neighbor sensing information base, the interface association set, and the topology set. Therefore, if any of such tables changes, the routing table has to be recalculated to update the available route information about each destination in the network. Basically, there are a number of possible routes between two communicating hosts, and each path may have a different available capacity, which may meet the QoS requirements of the desired service or not. Even if the selected path between a source-destination pair meets the user's needs at the session set-up time, the capacity and error characteristics observed along the path are susceptible to vary over time due to the multiple dynamics in the network. A regular verification is necessary since the link conditions may vary over time [8]. Hence, we can include the adaptive services feature, like a rerouting due to node mobility or after route degradation.

\subsection{The tailored metric for an Ad Hoc environment}

To improve the quality of service in such a network, it would be interesting include some parameters like delay, bandwidth, link cost, data loss and error rate. However, a well-known theorem in Constraint Based Routing [9] is that, computing optimal routes subject to constraints of two or more additive and/or multiplicative metrics is NP-complete [10].

Our proposal paper includes delay and bandwidth parameters to each route table entry corresponding to each destination in a suboptimal processing. However, one-way delay is traditionally the parameter most difficult to measure due to the need of time synchronization. This forces us to seek alternatives approximating the optimal solution. In order to achieve the delay information in the route calculation, a synchronized network is required. We suppose in our scenario that all the nodes are provided with a global timing structure. If, for example, GPS is used for this purpose, each node in the network receives clock ticks from a GPS receiver 
and uses them to keep its internal clock synchronized (i.e. there is a period of time in which the clocks of all the nodes have the same time value).

\subsection{Delay Metric}

In our proposal extension, a delay parameter will be calculated to improve the selection of the best path by the proposed routing algorithm.

Each node includes in the Hello message, during the neighbor discovery performed by the OLSR, the creation time of this message. As mentioned before, we suppose a synchronized network.

When the Hello message arrives in a neighbor node, the delay between the sender node and received node is calculated. The information about the neighborhood will be stored in the neighbor table, as proceeded in the OLSR standard, but in our proposal, we include the necessary delay to the sender node reaches the received node. Such procedure will be execute for all Hello messages without include any additional message to the routing protocol.

In order to obtain more accuracy, the delay value includes the variance in the statistical analysis, for example, if the actual delay is $19 \mathrm{~ms}$ and the last one was $1 \mathrm{~ms}$, the average delay is $10 \mathrm{~ms}$ but the variation between the values needs to be considered.

\subsection{Bandwidth Metric}

The bandwidth metric can specify the amount of bandwidth that will be available along a path from the source to the destination. Such information could be used by the OLSR protocol during the route calculation.

However, in a wireless environment, the bandwidth can vary as the node's mobility. In order to simplify the routing algorithm including the bandwidth metric, we intend to verify the bandwidth of the each channel only at the moment that the routing calculation is done. However, we forewarn that such measured bandwidth is not the overall bandwidth necessary to the path. Nevertheless, we can suppose as the OLSR routing protocol chooses routes always through MPR nodes, then in this case, the measured bandwidth met by the node should assist to select the best route.

Such information could be used in addition to the delay metric, to choose the best route for each node. The bandwidth information should be measured during the routing calculation, when the neighbor table and/or the topology table were changed. Such bandwidth calculation may not be the optimal, however it intends to be the simplest one.

\subsection{Admission control applied in each MPR}

An enhancement of the MPR concept is achieved by applying an admission control to the incoming traffic in each MPR node. We adopt an admission control mechanism to improve the overall performance of the system while assuring the routing of a new user's flow does not degrade the QoS provided to the current users. The admission control analyzes the available bandwidth to allow the selection of a MPR by a new node. The rule is based on the bandwidth average used in the MPR by the others nodes already chosen in this MPR. The threshold can be chosen in each node with information based on local measurements. Also a global threshold can be defined in order to preserve bandwidth for opening sessions. In this case, no more users are accepted until the system load becomes smaller.

In order to perform such functionality, we intend to use the new "willingness" field presented in the HELLO Message Format [4]. This field indicates the willingness of the sender to act as a multipoint relay. The willingness parameter is an integer between 0 and 7 . The value 0 is for nodes which should never forward packets to other destinations (e.g. because their power supply is critical, or in our case, if the threshold attaint $70 \%$ of the link capacity). The higher the willingness is, the higher the priority the node will have to be selected as relay. The value 7 is reserved for nodes which should be selected as multipoint relay in any case (e.g. when the node belongs to a pre-defined backbone). An OLSR router in normal operation should have willingness equal to 3, as specified in OLSR draft [4].

\section{PERformance EVAluAtion}

Our ongoing performance evaluation aims to assess the improvement achieved in QoS support capability by incorporating multi-criteria for routing decision to the original OLSR protocol which considers the number of hops criteria.

The analysis of network behavior, including new metrics in the proposed QoS-based routing and comparing with the OLSR standard protocol, must be achieved.

The aims of such performance evaluation is to verify the development of our proposed work performing the different scenarios and analyzing the evaluation results step by step. First, we implemented the QoS-based routing algorithm including only the delay metric in addition to the hop distance metric already used by the OLSR standard and afterwards we present the concluding results.

\subsection{Implemented Algorithm}

In order to achieve the evaluation performance for the addition of the delay metric in the OLSR protocol, we implement the next algorithm, represented in the schema below, and used in all studied scenarios. 


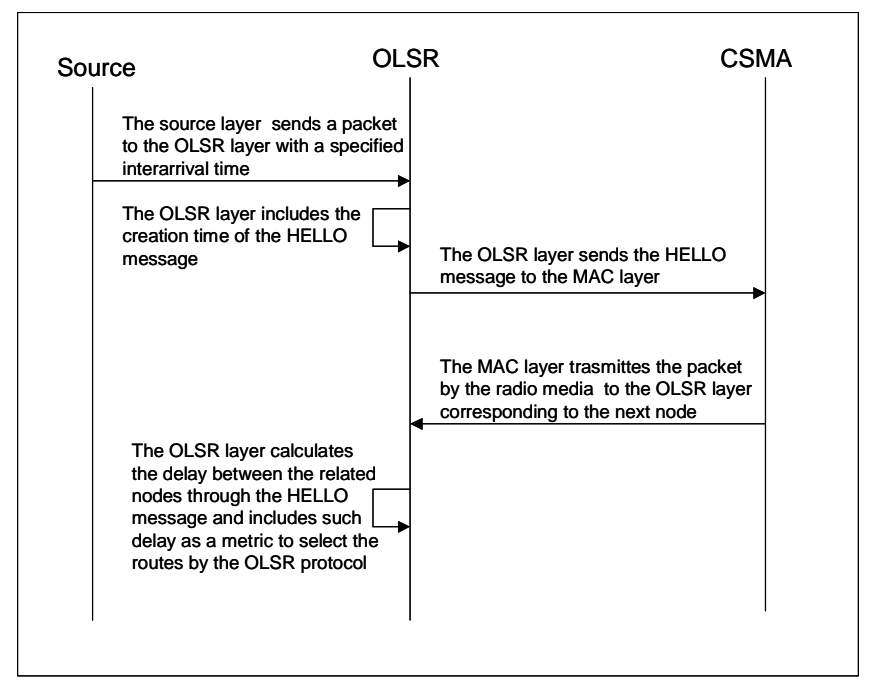

Figure 1. Generic schema implemented in our simulations.

We present some additional statements from our proposed algorithm:

- Before that the OLSR layer sends a hello message, it includes the time of the creation in the Hello packet.

- When the OLSR receives a hello message: gets the time stored in the hello message by the sender node; calculates the difference between such time and the current time, that represents the delay; and then calculates the average delay and the variance value to accuracy purposes

- During the routing calculation, if the sender node is a MPR, then the variance value will be tested. In this case, if the variance value were less than a defined threshold, in order to maintain the coherence of the used delay values, then such a route should set the weight variable as the average delay calculated. Else, the weight variable will receive, for instance, 1 second corresponding a less priority route.

In our simulation implementation of the standard OLSR, we use the well-known Dijkstra routing algorithm to route the packet using the number of hops metric. Such standard OLSR algorithm has a weight variable set in 1 due to the invariant weight among the routes.

However, we make use of such weight variable in order to implement different preference due to the delay information. After such weight variable will be used together with the number of hops to choose the best path to routing each packet.

We analyze only the value of MPR due to the OLSR routing mechanism always chose the MPR nodes to route the packets.

\subsection{Evaluation results}

Our simulation is performed by OPNET simulator [11]. We evaluate two different scenarios. The configured parameters in the simulated scenarios are: threshold variance is fixed in $10 \%$, the power transmission is 60 meters, the threshold of the reception is 0,00027 watts and the interarrival time is $60 \mathrm{~s}$.

In the first scenario, we perform the evaluation analyzing the average transmission time, varying the number of mobiles in a $100 \mathrm{~m} 2$ area network. For instance, we suppose the nodes without mobility, to after analyze all the possible results for the proposed protocol. We suppose the bandwidth equal to $10 \mathrm{~kb} / \mathrm{s}$.

The results found in such a scenario are shown in the following figure:

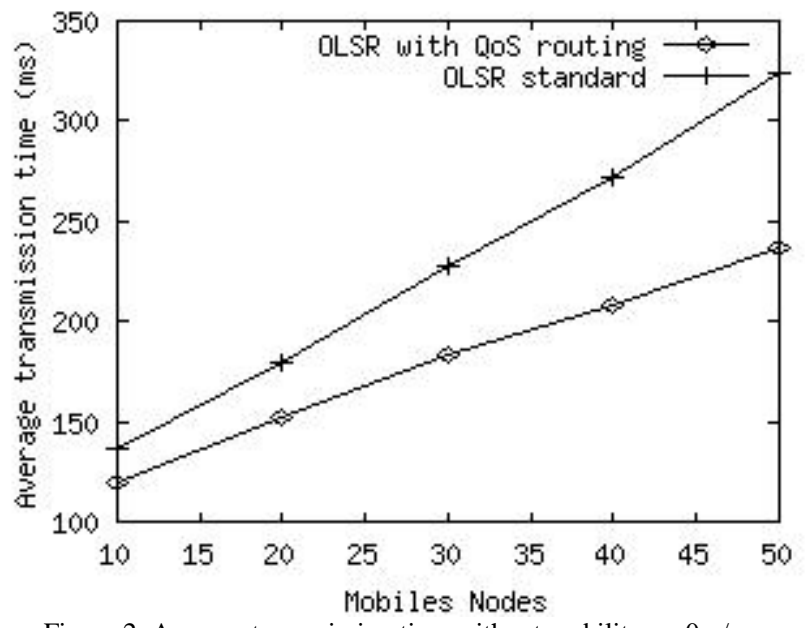

Figure 2. Average transmission time without mobility, v=0m/s.

The graphic presents an improvement comparing with the standard OLSR protocol to the average transmission time result, i.e. the complete time to a data packet leaves the sender node and arrives in the destination node. We can verify that as the number of the mobile nodes increase, the obtained gain is augmented.

The next evaluated scenario presents the comportment of the proposed QoS-based routing protocol supposing a network with a lot of collisions.

A better average transmission time is achieved by our proposed protocol as shown in figure 3. As the number of the mobiles increase, we can verify an attenuation of the average transmission time and an important gain achieved. 


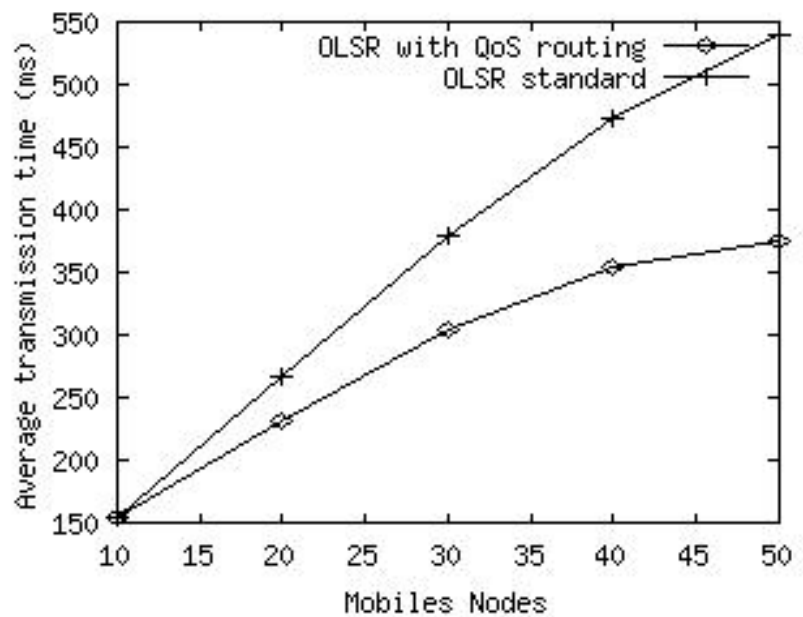

Figure 3. Average transmission time without mobility, $v=0 \mathrm{~m} / \mathrm{s}$ with a lot of collisions.

The presented results are very interesting, because of in a real wireless network, usually we have an important number of the collision due to the interferences of the radio transmission, and then, the obtained gain can improve the transmission time of such wireless networks.

We can also verify in the simulated scenarios an important gain obtained using our proposed QoS-based protocol. The obtained gain using our QoS-based routing protocol can reach $17,9 \%$ to the second scenario.

The next step aims to evaluate the system behavior varying the node mobility, for instance: $0 \mathrm{~m} / \mathrm{s}, 5 \mathrm{~m} / \mathrm{s}$ and $10 \mathrm{~m} / \mathrm{s}$.

After, we aim to include also the bandwidth metric to the proposed algorithm. We can expect that the necessary modification to include such metric should improve the gain obtained for our proposed algorithm due to the simple method used to improve the bandwidth metric proposed in section 3.3.

A next step will be the implementation of the proposed algorithm including the "willingness" field in the hello format, as proposed by the new OLSR draft [4] version 7. The actually OLSR protocol implementation is based on version 5 of the draft.

\section{CONCLUSION}

This paper presented a Link-state QoS Routing Protocol for Ad Hoc Networks. The proposal implements the QoS functionality to deal with limited available resources in a dynamic environment specifying a tailored metric for such a mobile wireless networks. In order to analyze the performance of the proposed QoS-based routing, we add some QoS values to the Optimized Link State Routing (OLSR) protocol, which already considers the hop distance, without include additional messages.
The presented evaluation performance estimated the improvement acquired by the proposed QoS-based routing protocol. The achieved gain by our proposal can be an important improvement in such mobile wireless networks.

We aim to proceed the performance evaluation implementing more scenarios and varying the simulation parameters, and also including the bandwidth metric and the admission control applied in the MPR nodes as proposed in this paper.

\section{REFERENCES}

[1] X. Xiao, L. M. Ni, "Internet QoS: A Big Picture", IEEE Network, Vol 13, No. 2, March/April 1999.

[2] S. Chakrabarti, A. Mishra, "QoS Issues in Ad Hoc Wireless Networks", IEEE Communications Magazine, February 2001.

[3] Elizabeth Royer and C-K Toh. "A review of current routing protocols for ad-hoc mobile wireless networks", IEEE Personal Communications Magazine, pages 46-55, April 1999. 23, 24.

[4] T. Clausen, P. Jacquet, A. Laouiti, P. Minet, P. Muhlethaler, A. Qayyum, and L. Viennot. "Optimized Link State Routing Protocol", IETF Internet Draft, draft-ietf-manet-olsr-07.txt.

[5] Chenxi Zhu, Scott Corson, "QoS routing for mobile ad hoc networks", IEEE Infocom 2002, New York, NY, June 23-27 2002.

[6] Charles E. Perkins, Elizabeth M. Belding-Royer, Samir R. Das, "Ad hoc On-Demand Distance Vector (AODV) Routing". In Internet-Draft, draft-ietf-manet-aodv-10.txt, January 2002.

[7] R. Sivakumar, P. Sinha, and V. Bharghavan, "CEDAR: a CoreExtraction Distributed Ad Hoc Routing Algorithm”, IEEE Infocom'99, New York, NY, March 1999.

[8] H. Xiao, W. K. G. Seah, A. Lo and K. C. Chua, "A Flexible Quality of Service Model for Mobile Ad-Hoc Networks", In the proceedings of IEEE Vehicular Technology Conference, 15-18 May 2000, Tokyo, Japan.

[9] E. Crawley, R. Nair, B. Jajagopalan and H. Sandick, "A Framework for QoS-based Routing in the Internet", RFC 2386, Aug. 1998.

[10] Z. Wang and J. Crowcroft, "Quality of Service Routing for Supporting Multimedia Applications", IEEE JSAC, Sept. 1996.

[11] OPNET simulator, http://www.opnet.com/ 